\title{
Short-term implicit memory: Visual, auditory, and cross-modality priming
}

\author{
ELINOR MCKONE and CHRISTOPHER DENNIS \\ Australian National University, Canberra, Australia
}

\begin{abstract}
Short-term implicit memory was examined for mixed auditory (A) and visual (V) stimuli. In lexical decision, words and nonwords were repeated at lags of $0,1,3$, and 6 intervening trials, in four primetarget combinations (VV, VA, AV, AA). Same-modality repetition priming showed a lag $\times$ lexicality interaction for visual stimuli (nonwords decayed faster), but not for auditory stimuli (longer lasting smooth decay for both words and nonwords). These modality differences suggest that short-term priming has a perceptual locus, with the phonological lexicon maintaining stimuli active longer than the orthographic lexicon and treating pseudowords as potential words. We interpret these differences in terms of the different memory needs of speech recognition and text reading. Weak cross-modality short-term priming was present for words and nonwords, indicating recoding between perceptual forms.
\end{abstract}

Long-lived repetition priming occurs on various word recognition tasks. Word-nonword decision times are faster for repeated items in lexical decision (Kirsner \& Smith, 1974), as are reading-aloud times in naming (Monsell, 1985), and the repetition benefit can endure for minutes or days with only slow decay (e.g., McKone, 1998). We refer to this type of priming as long-term implicit memory, where implicit memory refers to automatic retrieval of the target's initial presentation and is contrasted with the deliberate conscious retrieval required for explicit memory revealed in recall and oldnew recognition tasks (Graf \& Schacter, 1985). Recently, McKone (1995; also see Maljkovic \& Nakayama, 1994) identified an additional short-lived component of repetition priming, with a duration of approximately three items $(8 \mathrm{sec})$ for visually presented words in lexical decision and naming. We refer to this effect as short-term implicit memory, where the label short-term was chosen to reflect its similarity in duration to that of standard explicit short-term or "working" memory (Baddeley \& Hitch, 1974). We do not assume that different stores subserve short- and long-term priming. Our preferred theoretical interpretation is of two mechanisms operating within a common system: In a connectionist approach, for example, short-term priming might reflect transient activation of units, whereas long-term priming might reflect changes in weights on the connections between these same units.

McKone (1995) examined priming for written words and nonwords (pseudowords like sime) at various numbers of intervening trials in a continuous sequence. For

This research was supported by Grant F96058 from the Australian Research Council. We thank Daniel Schacter and Erik Bergman for comments on a previous version of this article. Correspondence should be addressed to E. McKone, Division of Psychology, Australian National University, ACT 0200, Australia (e-mail: elinor.mckone@anu.edu.au). words in lexical decision, the repetition advantage at lag 0 (immediate repeat) was $100 \mathrm{msec}$ greater than the stable long-term value reached after lag 4 , and priming decayed with a smooth, approximately exponential form across lags $0,1,2$, and 3 . Word frequency influenced the long-term priming component only. For high-frequency words, the $100 \mathrm{msec}$ of short-term priming existed in the absence of any priming at all beyond lag 4, whereas for low-frequency words, the $100 \mathrm{msec}$ of short-term priming was additional to a long-term component of $45 \mathrm{msec}$ (i.e., total priming at lag 0 was $145 \mathrm{msec}$ ). Thus, the word frequency manipulation dissociated short-term implicit memory from long-term implicit memory. It also indicated that total priming at short lags reflects the sum of contributions from both components.

For nonwords, in contrast to words, an initial $100 \mathrm{msec}$ of short-term priming decayed precipitously to the longterm value with only a single intervening item. This produced a lag $\times$ lexicality interaction, with words and nonwords differing most strongly at lags 1 and 2 . This pattern was found in both lexical decision and naming. Changing the task to explicit recognition (old-new decision), however, produced slower overall responses and destroyed the lag $\times$ lexicality interaction, leading to smooth decay over short lags for both item types. Thus, the lexical status manipulation dissociated short-term implicit memory from short-term explicit memory.

The lack of a lexicality effect on short-term explicit memory is consistent with the idea that verbal rehearsal in an articulatory loop (Baddeley \& Hitch, 1974) is possible for both words and pseudowords. In contrast, the lag $\times$ lexicality interaction in lexical decision and naming argues that short-term implicit memory more directly reflects transient traces within the word recognition system itself. We hypothesize that the function served by such an implicit "working memory" is to support the integration over time of discrete items, such as words in sentences, in subconscious processing. 
Given that words and pseudowords contain the same visual patterns and the same letters, the lag $\times$ lexicality interaction must arise from traces left at a lexical level of representation (where lexical can include either localized or distributed word representations) and not from a more peripheral sensory locus. Neuropsychological evidence indicates that there are at least three modules of the word recognition system which contain lexical representations (Coltheart, 1989). Two of these are perceptual in nature, namely the orthographic lexicon (for written words; Schacter, 1994, calls this the visual word form system) and the phonological lexicon (for spoken words). The third is a semantic lexicon storing word meaning. The detailed explanations of short-term priming patterns that we have proposed elsewhere (McKone, 1995,1998 ) have assumed an orthographic locus, given that stimuli were presented visually. This assumption reflects a "perceptual" view of priming on tasks such as lexical decision and naming (as has been proposed for long-term priming, e.g., Schacter, 1994). However, we have no direct evidence that the locus for written stimuli is specifically orthographic: Words and pseudowords also differ in their phonological and semantic familiarity status.

The present experiment, therefore, tested the perceptual view of short-term priming, using mixed auditory (A) and visual (V) stimuli. Both same-modality priming (VV and AA prime-target combinations) and crossmodality priming (VA and $\mathrm{AV}$ ) were of interest. If the locus of short-term priming is the relevant perceptual module, then same-modality priming for auditory stimuli should rely on traces within the phonological lexicon, while priming for visual stimuli should be based in the orthographic lexicon. The different requirements of speech recognition and reading suggest that the shortterm memory needs of these two processing modules might be rather different. Spoken-word signals arrive spread out over time, while written-word signals are presented in parallel, suggesting overall duration differences. More interestingly, continuous speech is highly ambiguous in that there are no clear breaks between words and there is no one-to-one correspondence between a segment of speech signal and word identity (Pollack \& Picket, 1964), while written text (for typewriting at least) is far less ambiguous. This suggests the phonological lexicon might treat words and pseudowords more similarly than does the orthographic lexicon, and thus that different patterns of AA and VV short-term priming might appear, particularly for nonwords.

Turning to cross-modal priming, a perceptual locus would predict no short-term priming for either words or nonwords if it is assumed that short-term priming relies solely on traces within the relevant input perceptual module. Alternatively, some limited cross-modal shortterm priming might occur, if activation in the input module automatically produces a corresponding pattern of activation in the other perceptual subsystem (e.g., written words and pseudowords undergo obligatory recoding to their spoken form). If short-term priming is not perceptual at all (e.g., it relies on central semantic representations accessed by both input modalities, or it reflects explicit memory), one of two patterns might appear: complete cross-modal transfer of priming for real words with no cross-modal priming at all for nonwords, or complete cross-modal transfer for all stimuli.

Only one previous study has examined priming at short lags as a function of both lexical status and modality. Kirsner and Smith (1974) tested lags 0, 3, 15, and 63 in lexical decision. They found general patterns of a reduction in priming at longer lags, and smaller crossmodality than same-modality priming. However, their analysis did not directly compare priming patterns for $V V$ versus $A A$, and made no distinction between a shortand a long-term component of priming. Critically, they did not test lag 1 or 2, which is where McKone (1995) found visual words and nonwords to differ most strongly. Our experiment was similar to that of Kirsner and Smith, but specifically targeted the region in which the lag $X$ lexicality interaction is found with visual presentation. We also increased the number of items per condition to 20 (they used 10 ), fully randomized trial order and the selection of items into conditions (they used only two different versions of the list), and used a more appropriate calculation of the baseline for lag 0 priming conditions (see the Results section).

\section{METHOD}

\section{Subjects}

Subjects were 22 volunteers with English as their first language. Most were undergraduate psychology students.

\section{Design}

All manipulations were within subjects. Lexical status (word, nonword) was crossed with first presentation modality (auditory, visual), second presentation modality (auditory, visual), and lag $(0$, 1,3 , or 6 items intervening between repeats).

\section{Materials}

Stimuli were four-letter, single-syllable words and pseudowords (nonwords satisfying the rules of English, e.g., sime). The 320 target words were low frequency (1-15 per million; Kučera \& Francis, 1967) and excluded proper nouns, extremely unfamiliar words, and words with ambiguous translation from orthography to phonology (e.g., wind) or vice versa (e.g., reed/read), so it was clear which word was being primed in the cross-modality conditions. The 320 target nonwords excluded homophones of real words (e.g., kean) or of any other nonwords in the list (e.g., cabe and kabe were not both included). Ambiguity in orthographic-phonological translation was limited as much as possible by asking 6 pilot subjects to either spell each nonword from dictation or to pronounce the written form. Items producing any disagreement were replaced.

The list for each subject comprised 1,500 trials in five blocks of 300 . There were 32 priming conditions (see Design section) with 20 items in each. Targets were repeated, so 1,280 trials presented all conditions of interest ( 32 conditions $\times 20$ items $\times 2$ presentations). 
The remaining 220 trials contained unrepeated fillers ( 110 words and 110 pseudowords). Overall, $43 \%$ of trials were repeats of previous items.

Four occurrences of each priming condition appeared in each of the five blocks to avoid gross differences in average position within the list. No repeats occurred across blocks. To minimize the length of the list, lag conditions were closely overlapped within blocks, such that target items for one condition were often intervening items for others. The order of priming conditions was rerandomized for every block for every subject. Across 22 subjects and 20 occurrences per condition, 440 different random orderings of trials led up to the presentation of any given condition. The selection of 20 items into each lag/modality condition was also rerandomized for each subject. Thus, there should be no systematic bias in the priming data due to trial order or items assigned to each condition.

\section{Procedure}

Subjects were tested individually in a $90-\mathrm{min}$ session. There were 30 practice trials. Each experimental block took $10 \mathrm{~min}$. Stimul were presented on a PowerMac Macintosh computer. Auditory stimuli were recorded in a male voice using SoundEdit software, and played to subjects over earphones. Visual stimuli were presented at the center of the screen in lowercase. Lexical decision times were measured accurate to $1 \mathrm{msec}$ using a Button Box read by PsyScope software (Cohen, MacWhinney, Flatt, \& Provost, 1993).

A new trial began every $2 \mathrm{sec}$. Visual stimuli were cleared from the screen when the subject responded. Auditory stimuli were played until the speech signal was complete, and the remainder of the $2 \mathrm{sec}$ was silent. Subjects pressed one button to respond "word" and another for "nonword." Reaction time (RT) was recorded from the onset of the stimulus. Subjects were told to respond as quickly and accurately as possible. No feedback was given.

\section{RESULTS}

Lexical decision times were excluded if they fell below 300 or above $1,700 \mathrm{msec}(1.3 \%$ of RTs), or if an incorrect response was given to either presentation of the item $(6.2 \%$ of RTs $)$.

\section{Baselines for Priming}

Unprimed responses were much faster to visual stimuli than to auditory stimuli (words: $\mathrm{V}=620 \mathrm{msec}, \mathrm{A}=$ 966 msec; nonwords: $\mathrm{V}=688 \mathrm{msec}, \mathrm{A}=1,007 \mathrm{msec}$ ). Thus, priming cannot be taken as the difference in RTs between the first and second presentations of the same item, particularly in the cross-modal conditions. Instead, we followed the procedure of Kirsner and Smith (1974) in choosing the baseline for priming to be the mean of all unprimed trials of the appropriate lexicality and modality. For example, priming in the lag $1 \mathrm{AV}$ word condition was calculated as the mean RT to second presentations in this condition, subtracted from the mean RT to first presentations of all visually presented words (i.e., $620 \mathrm{msec}$ ).

This approach provides a suitable method of calculating the advantage arising from item repetition at lags 1 , 3 , and 6 . There is a problem at lag 0 , however, which was not recognized by Kirsner and Smith (1974). A post hoc split of our data showed that first presentation RTs were consistently faster to trials that matched the preceding trial in modality. For visual words (nonwords in brack- ets), mean unprimed RTs were $595 \mathrm{msec}(661 \mathrm{msec})$ if the preceding trial was also visual, but $646 \mathrm{msec}(714 \mathrm{msec})$ if the preceding trial was auditory. For auditory words (nonwords), RTs were $946 \mathrm{msec}(999 \mathrm{msec})$ if the preceding trial was also auditory, but $984 \mathrm{msec}(1,015 \mathrm{msec})$ if the preceding trial was visual.

This affects the calculation of baselines for lag 0 repeats, because primed trials in these conditions were preceded by a fixed modality for all 20 occurrences (e.g., for lag $0 \mathrm{AV}$, the trial preceding the primed $\mathrm{V}$ presentation was always auditory). In contrast, unprimed first presentations could be preceded by either modality (e.g., in each of the VV or VA conditions, on average only $50 \%$ of the trials preceding the unprimed $V$ presentation were auditory). This results in a confounding of any repetition advantage with effects of prior modality if all first presentations are used as the baseline for priming. Instead, the baselines used for lag 0 priming were the values given in the previous paragraph: For example, second presentation RTs in the lag $0 \mathrm{AV}$ word condition were compared with the mean RT to first presentations of visual words preceded by auditory trials (i.e., 646 not $620 \mathrm{msec}$ ).

\section{Same-Modality Short-Term Priming: Visual Versus Auditory}

Figure 1 shows repetition priming for words and nonwords as a function of lag in the two same-modality conditions (VV, AA). Figure la shows that when the stimu-

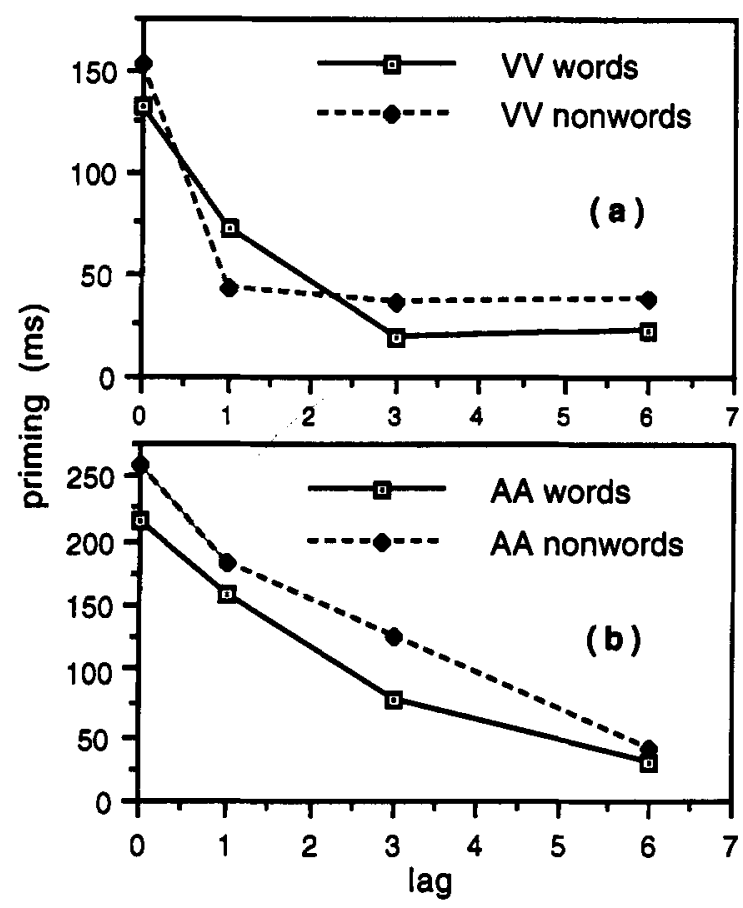

Figure 1. Same-modality repetition priming for low-frequency words and pseudowords as a function of lag, for (a) visual (V) stimuli and (b) auditory (A) stimuli. Note the different $y$-axis scales. 
lus appeared in written form on both presentations, the same patterns reported with visual presentation in McKone (1995) were evident. That is, short-term priming (priming above the stable long-term value) endured longer for words than for nonwords. A repeated measures two-way analysis of variance (ANOVA) confirmed a significant lag $\times$ lexicality interaction $[F(3,63)=2.76$, $\left.M S_{\mathrm{e}}=2,416, p<.05\right]$. Helmert contrasts confirmed its form. For words $\left(M S_{\mathrm{e}}=2,531\right)$, priming at lag 0 was significantly above the mean of all later lags $[F(1,63)=$ $31.4, p<.001]$, and priming at lag 1 remained significantly above the mean of lags 3 and $6[F(1,63)=15.4$, $p<.001]$. By lag 3 , word priming was not significantly different from that at lag $6(F<1)$. For nonwords $\left(M S_{\mathrm{e}}=\right.$ 1,872 ), however, priming was above the average of later lags only at lag $0[F(1,63)=112.7, p<.001]$. Priming dropped to the long-term value with a single intervening item, with no significant difference between lag 1 and the average of lags 3 and $6(F<1)$ or between lag 3 and lag $6(F<1)$.

Figure $1 \mathrm{~b}$ shows a very different pattern of short-term priming for when both presentations of the stimulus were auditory. Two effects are apparent: Short-term priming was larger and longer lasting than with written presentation, and nonword priming now decayed in the same manner as word priming. A two-way ANOVA yielded no suggestion of a lag $\times$ lexicality interaction $\left(F<1, M S_{\mathrm{e}}=\right.$ $5,327)$. Helmert contrasts demonstrated short-term priming to lag 3 for both words $\left(M S_{\mathrm{e}}=4,556\right)$ and nonwords $\left(M S_{\mathrm{e}}=6,069\right)$. Priming at lag 0 was significantly above the mean of all later lags both for words $[F(1,63)=$
$57.6, p<.001]$ and for nonwords $[F(1,63)=55.5, p<$ $.001]$. Priming at lag 1 was above the mean of lags 3 and 6 both for words $[F(1,63)=34.4, p<.001]$ and nonwords $[F(1,63)=24.4, p<.001]$. Priming at lag 3 remained above that at lag 6 for both words $[F(1,63)=5.52, p<$ $.05]$ and nonwords $[F(1,63)=12.94, p<.001]$. It is even possible that short-term priming with auditory presentation extended beyond lag 3 . Priming at lag 6 reached the 20-50 msec range, at which we might expect long-term priming to stabilize for low-frequency words, but the inclusion of no longer lags means that there is no direct evidence that the long-term value was attained by lag 6 .

\section{Cross-Modality Short-Term Priming}

Figure 2 compares priming in the cross-modality conditions (AV, VA) with that in the relevant same-modality condition. All four panels of this figure show that shortterm priming in the cross-modality conditions was present (i.e., priming was above the long-term value), but was reduced relative to same-modality priming (i.e., it was not as far above the long-term value). For example, Figure 2a shows that for words repeated visually, shortterm priming was greater when the first presentation was also visual (VV words) and was reduced when the first presentation was auditory (AV words). Some short-term priming remained, however, even with the cross-modal prime. Two-way ANOVAs revealed significant interactions between lag and same- versus cross-modality priming conditions in all cases: for visual words $\left[F(3,63)=2.90, M S_{\mathrm{e}}=\right.$ $2,443, p<.05]$, for auditory words $\left[F(3,63)=3.20, M S_{\mathrm{e}}\right.$ $=3,734, p<.05]$, for visual nonwords $\left[F(3,63)=7.6, M S_{\mathrm{e}}=\right.$

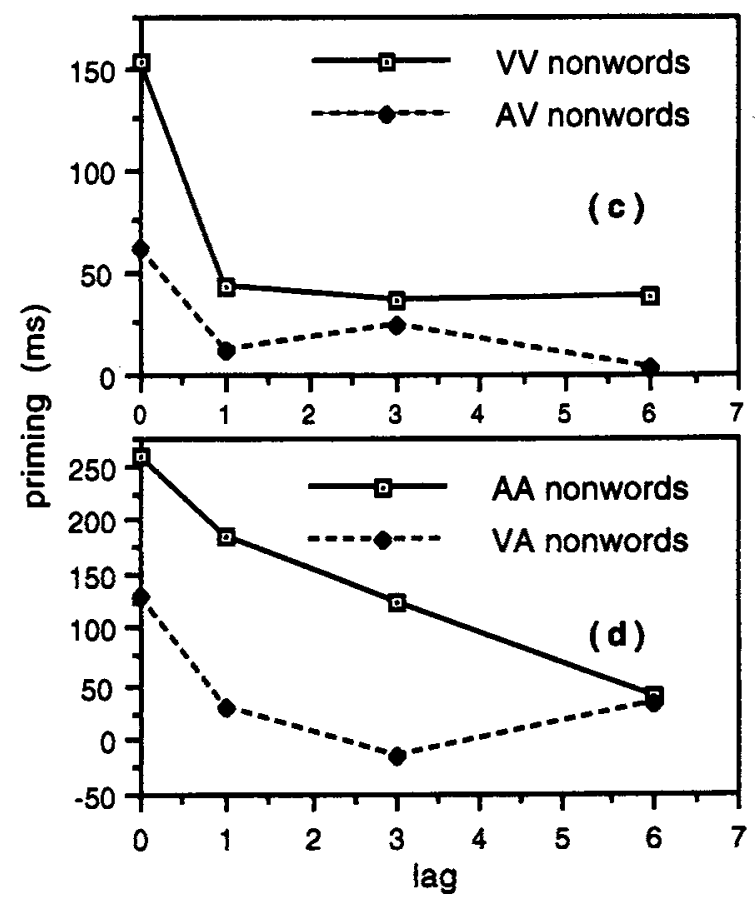

Figure 2. Same- versus cross-modality priming, where the primed trials were (a) visual (V) words, (b) auditory (A) words, (c) visual nonwords, and (d) auditory nonwords. 
$1,705, p<.001]$, and for auditory nonwords $[F(3,63)=$ 8.6, $\left.M S_{\mathrm{e}}=5,757, p<.001\right]$.

Helmert contrasts were used to confirm the existence of some short-term priming in the cross-modal conditions and to track the form of its decay. For AV words (Figure $2 \mathrm{a} ; M S_{\mathrm{e}}=2,815$ ), there was significantly more priming at lag 0 than at all later lags $[F(1,63)=22.6, p<$ $.001]$. The priming advantage at lag 1 over the two later lags did not achieve significance $[F(1,63)=2.58, p>.1]$; nor did the lag 3 advantage over lag $6(F<1)$. A direct comparison of lags 1 and 6 did, however, demonstrate decay $[F(1,63)=4.26, p<.05]$. For VA words (Figure $2 \mathrm{~b} ; M S_{\mathrm{e}}=4,633$ ), priming was above the later lags at lag $0[F(1,63)=56.7, p<.001]$ and at lag $1[F(1,63)=$ $7.69, p<.01]$. Lags 3 and 6 did not differ $(F<1)$. For nonwords, AV nonwords (Figure $2 \mathrm{c} ; M S_{\mathrm{e}}=1,874$ ) showed more priming at lag 0 than at later lags $[F(1,63)=$ 20.3, $p<.001]$. Priming did not differ between lag 1 and later lags $(F<1)$ or between lags 3 and $6[F(1,63)=2.64$, $p>.1]$. For VA nonwords (Figure $2 \mathrm{~d} ; M S_{\mathrm{e}}=6,194$ ), lag 0 priming was above later lags $[F(1,63)=42.5, p<.001]$, while lag 1 priming was not $[F(1,63)=1.27, p>.1]$, and lag 3 priming even fell significantly below the lag 6 value $[F(1,63)=4.78, p<.05]$.

Thus, all cross-modal conditions revealed significant short-term priming at lag 0 , although this was smaller than in the same-modal condition. Short-term crossmodal priming endured to lag 1 when the target was a word, but cross-modal priming for nonwords dropped to the long-term value with a single intervening item. These patterns were found regardless of the modality of the second presentation.

\section{Cross-Modality Long-Term Priming}

Although the focus of this experiment was on shortterm priming, we also examined the effects of modality mismatch on long-term priming, as reflected by priming at lag 6. Figure 2 shows much weaker cross-modality than same-modality long-term priming for visual targets (Figures $2 \mathrm{a}, 2 \mathrm{c}$ ), but only a small reduction for auditory targets (Figures 2b, 2d). A three-way ANOVA on the lag 6 data confirmed an interaction between modality of first presentation and modality of second presentation $\left[F(1,21)=5.08, M S_{\mathrm{e}}=3,033, p<.04\right]$, with no interactions involving lexical status (all $F \mathrm{~s}<1$ ). Collapsing over lexicality, the cross-modal reduction in priming was significant for visual targets [ $31 \mathrm{vs} .2 \mathrm{msec}, t(21)=3.57$, $p<.005$ ], but not for auditory targets (36 vs. $27 \mathrm{msec}$, $t<1)$. For visual targets, the significant reduction replicates many similar findings in the long-term priming literature, in both lexical decision (e.g., Kirsner, Milech, \& Standen, 1983) and other implicit memory tasks (see Roediger \& McDermott, 1993, for review). Few studies have used auditory targets, but we note that our small effect appears to conflict with Kirsner and Smith's (1974) large cross-modal reduction in long-term priming in lexical decision ( 83 vs. $-14 \mathrm{msec}$, for words at lags 15 and 63).

\section{Error Rates}

Error rates dropped from $11.3 \%$ on first presentation to $6.0 \%$ on second presentation for words, and from $5.9 \%$ to $5.7 \%$ for nonwords. Thus, speed-accuracy tradeoffs were not responsible for priming in RTs. Effects of the independent variables on error priming were also tested. A four-way ANOVA revealed no significant interactions (alpha $=.05$ ). Three main effects were found: More priming occurred for words than for nonwords $(5.3 \%$ vs. $0.2 \%)$, for visual primes than for auditory primes $(3.7 \%$ vs. $1.9 \%)$, and for shorter lags than for longer lags $(\operatorname{lag} 0=4.3 \%$, lag $1=3.4 \%$, lag $3=1.5 \%$, $\operatorname{lag} 6=2.9 \%$ )

\section{DISCUSSION}

The findings regarding short-term priming were as follows. First, the results of McKone (1995) were replicated when both presentations were visual, although the list used here contained mixed auditory and visual stimuli. Decay was somewhat more rapid, but the key lag $\times$ lexicality interaction was found. Second, short-term auditory priming was shown to be longer lasting than short-term visual priming. Third, the increased duration for auditory items was particularly striking for nonwords: whereas visual presentation produced decay to the long-term value with a single intervening item, auditory presentation produced a short-term priming effect for nonwords that endured at least three intervening trials $(8 \mathrm{sec})$ and decayed in exactly the same manner as for words. Fourth, shortterm priming was reduced by modality shift, but did not disappear completely; this general pattern was observed for both words and nonwords.

Our finding of stronger short-term priming with auditory than with visual presentation parallels similar results obtained when short-term memory is assessed with explicit, rather than implicit, retrieval tasks. An auditory presentation advantage in short-term explicit memory has been obtained for both words and nonsense syllables (see Penney, 1989, for review), and previous authors have made a general link between this advantage and the temporal extent of auditory signals. The most striking aspect of our results in short-term implicit memory, however, was that the lag $\times$ lexicality interaction occurs with visual presentation but not with auditory presentation. For explicit short-term memory, we know of no studies directly comparing lexicality effects in each modality, although we note that McKone (1995) found no lag $\times$ lexicality interaction with visual presentation in old-new recognition. Thus, it appears that the differential effects of lexicality in the two modalities are more striking with implicit than explicit tests. This might be expected if implicit tasks rely more directly on modalityspecific perceptual processes rather than on verbal rehearsal in a common articulatory loop.

The very different patterns of short-term priming for visual and auditory stimuli are consistent with our view that such priming arises from short-lived traces main- 
tained within the perceptual subsystems of word recognition. We propose that an explanation of the AA and VV patterns, and particularly the different effects of lexical status in each modality, might lie in the relative signal-to-identity ambiguity of spoken and written words. If short-term priming is the result of a form of implicit working memory, it presumably reflects the temporary holding of each stimulus as "active" so that it can be integrated with successive items (e.g., in sentence processing). This process is much more critical for speech perception than for reading typewritten text. In continuous speech, the segment of signal corresponding to a single word often cannot be understood in isolation, and several to many words of surrounding context are required (Pollack \& Picket, 1964). That is, the speech signal corresponding to a real word initially is not discriminable from a nonword and, to give the stimulus its correct interpretation, must be held until several successive speech segments are processed. The phonological lexicon, therefore, has need to hold items assigned pseudoword status active for as long as those assigned word status: In continuous speech, many "nonwords" will later turn out to be real words. With (type)written presentation, the signal is much less ambiguous. The orthographic lexicon can afford, therefore, to rapidly discard a nonword when the next item is presented.

Our results from the same-modality conditions indicate that the primary locus for short-term priming is the perceptual subsystem to which the stimulus is presented (i.e., orthographic lexicon for $\mathrm{V}$, phonological lexicon for A). The finding of partial cross-modality priming for both words and nonwords, however, indicates that this presentation module is not the sole locus. The results suggest that each stimulus undergoes obligatory recoding into the alternative perceptual form (i.e., orthographic to phonological and, more interestingly, vice versa), and that a secondary trace of this recoding can contribute to short-term priming. This conclusion remains consistent with the general idea that short-term priming relies on traces left within perceptual subsystems, rather than on more central traces (e.g., a semantic locus would have predicted complete cross-modal short-term priming for words and none at all for pseudowords).

Finally, our results confirm that the lag $\times$ lexicality interaction is not a methodological artifact of the opposite decisions made to words and nonwords in lexical decision. With written stimuli, McKone (1995) found the interaction in naming as well as in lexical decision. Here, it disappeared in lexical decision when the input modality was auditory. McKone (1998) has shown that the interaction in fact arises from a particular sensitivity of visual nonword traces to interference from intervening items, a conclusion that is consistent with the present proposed explanation of the differences between auditory and visual short-term priming.

\section{REFERENCES}

BAdDElEy, A. D., \& Hitch, G. (1974). Working memory. In G. H. Bower (Ed.), The psychology of learning and motivation (Vol. 8, pp. 47-90). New York: Academic Press.

Cohen, J., MacWhinney, B., Flatt, M., \& Provost, J. (1993). PsyScope: An interactive graphic system for designing and controlling experiments in the psychology laboratory using Macintosh computers. Behavior Research Methods, Instruments, \& Computers, 25, 257-271.

COLTHEART, M. (1989). Implicit memory and the functional architecture of cognition. In S. Lewandowsky, J. C. Dunn, \& K. Kirsner (Eds.), Implicit memory: Theoretical issues (pp. 285-297). Hillsdale, NJ: Erlbaum.

GraF, P., \& Schacter, D. L. (1985). Implicit and explicit memory for new associations in normal and amnesic subjects. Journal of Experimental Psychology: Learning, Memory, \& Cognition, 11, 501. 518.

KIRSNer, K., Milech, D., \& StAnden, P. (1983). Common and modalityspecific processes in the mental lexicon. Memory \& Cognition, 11, $621-630$.

KIRSNER, K., \& SMITH, M. C. (1974). Modality effects in word identification. Memory \& Cognition, 2, 637-640.

KuČERA, M., \& FranCIS, W. (1967). Computational analysis of presentday American English. Providence, RI: Brown University Press.

Maljkovic, V., \& NaKayama, K. (1994). Priming of pop-out: I. Role of features. Memory \& Cognition, 22, 657-672.

MCKoNE, E. (1995). Short-term implicit memory for words and nonwords. Journal of Experimental Psychology: Learning, Memory, \& Cognition, 21, 1108-1126.

MCKone, E. (1998). The decay of short-term implicit memory: Unpacking lag. Memory \& Cognition, 26, 1173-1186.

MONSELL, S. (1985). Repetition and the lexicon. In A. W. Ellis (Ed.), Progress in the psychology of language (pp. 147-195). London: Erlbaum.

PENNEY, C. G. (1989). Modality effects and the structure of short-term verbal memory. Memory \& Cognition, 17, 398-422.

POLLACK, I., \& PiCKET, J. M. (1964). The intelligibility of excerpts from conversational speech. Language \& Speech, 6, 165-171.

RoEDIGER, H. L., III, \& MCDERMOTT, K. B. (1993). Implicit memory in normal human subjects. In F. Boller \& J. Grafman (Eds.), Handbook of neuropsychology (Vol. 8, pp. 63-131). Amsterdam: Elsevier.

SCHACTER, D. L. (1994). Priming and multiple memory systems: Perceptual mechanisms of implicit memory. In D. L. Schacter \& E. Tulving (Eds.), Memory systems 1994 (pp. 233-268). Cambridge, MA: MIT Press, Bradford Books.

(Manuscript received April 15, 1997; revision accepted for publication June 28,1999 .) 